\title{
Transcriptional profiling of the peripheral blood response during tularemia
}

\author{
H Andersson ${ }^{1}$, B Hartmanová1,3, E Bäck², H Eliasson², M Landfors ${ }^{1}$, L Näslund ${ }^{1}$, P Rydén ${ }^{1}$ \\ and A Sjöstedt ${ }^{1}$ \\ ${ }^{1}$ Department of Clinical Microbiology, Clinical Bacteriology, Umeå University, Umeå, Sweden; ${ }^{2}$ Department of Infectious Diseases, \\ Örebro University Hospital, Örebro, Sweden and ${ }^{3}$ Faculty of Military Health Science, Proteome Center for the Study of Intracellular \\ Parasitism of Bacteria, University of Defence, Hradec Králové, Czech Republic
}

\begin{abstract}
Tularemia is a febrile disease caused by the highly contagious bacterium Francisella tularensis. We undertook an analysis of the transcriptional response in peripheral blood during the course of ulceroglandular tularemia by use of Affymetrix microarrays comprising 14500 genes. Samples were obtained from seven individuals at five occasions during 2 weeks after the first hospital visit and convalescent samples 3 months later. In total, 265 genes were differentially expressed, 95 of which at more than one time point. The differential expression was verified with real-time quantitative polymerase chain reaction for 36 genes $\left(R^{2}=0.590\right)$. The most prominent changes were noted in samples drawn on days 2-3 and a considerable proportion of the upregulated genes appeared to represent an interferon- $\gamma$-induced response and also a proapoptotic response. Genes involved in the generation of innate and acquired immune responses were found to be downregulated, presumably a pathogen-induced event. A logistic regression analysis revealed that seven genes were good predictors of the early phase of tularemia. This is the first description of the transcriptional host response to ulceroglandular tularemia and the study has identified gene subsets relevant to the pathogenesis of the disease and subsets that may serve as early diagnostic biomarkers.
\end{abstract}

Genes and Immunity (2006) 7, 503-513. doi:10.1038/sj.gene.6364321; published online 6 July 2006

Keywords: tularemia; host response; microarray; IFN- $\gamma$-induced; diagnostic markers

\section{Introduction}

Genome-wide gene expression profiling has been used to better classify many cancers and to understand the molecular pathways involved in several inflammatory disease processes. A number of publications have identified host signatures expressed during infectious diseases. For example, the molecular and cellular features of hemorrhagic smallpox in macaques have been analyzed by use of cDNA microarrays and certain unique features were identified. ${ }^{1}$ In a murine model of sepsis, the transcriptional response of peripheral blood cells (PBC) was undertaken. ${ }^{2}$ Some $5 \%$ of assessed genes were differentially regulated and most of the genes were present in both Gram-negative and Gram-positive sepsis. Thus, the implementation of the large-scale microarray technique is a powerful tool for elucidating the specific host adaptation to infection.

Tularemia is a disease caused by the highly contagious bacterium Francisella tularensis. It characteristically presents as an acute illness with nonspecific symptoms such as chills, fever, malaise and headache. The disease can be

Correspondence: Professor A Sjöstedt, Department of Clinical Bacteriology, Umeå University, SE-901 85 Umeå, Sweden.

E-mail: Anders.Sjostedt@climi.umu.se

Received 1 February 2006; revised and accepted 30 May 2006; published online 6 July 2006 acquired via several routes and the clinical presentation depends on the route of entry (reviewed by Tarnvik et al. ${ }^{3}$ ). Inhalation of $F$. tularensis subsp tularensis gives rise to the most aggressive form of tularemia, the respiratory form that in both animals and humans is associated with considerable mortality. ${ }^{4}$ Transmission by arthropods causes the ulceroglandular form of the disease and it may also result after direct contact with infected animals. A third, rather rare form is oropharyngeal tularemia, which occurs after oral ingestion of contaminated food or water. Another unusual form of the disease is the oculoglandular, which leads to conjunctivitis and photophobia.

In European countries, the ulceroglandular form caused by the $F$. tularensis subsp holarctica is the predominant variant of the disease and in Sweden, it constitutes $>90 \%$ of the cases. ${ }^{3}$ At the site of infection, a primary ulcer develops, often from a papule into a pustule. ${ }^{5}$ The ulcer is sometimes inconspicuous and usually heals within a week and can be mistaken for an arthropod bite. Although the ulcer heals, the draining lymph nodes enlarge, become palpable and tender, and if proper antibiotic treatment is not instituted within the first 2 weeks of infection, severe enlargement and sometimes suppuration ensues. Besides these clinical manifestations, little is known about other aspects of the disease, for example, if it results in the spread of $F$. tularensis to spleen and liver. Generally, changes in 
peripheral blood are inconspicuous as evidenced by small increases in mean leukocyte counts and, moreover, mean peak C-reactive protein values are remarkably low for an invasive disease. ${ }^{6}$ To better understand the pathogenesis of the disease and some of the rather paradoxical features of ulceroglandular tularemia, we performed a large-scale microarray analysis of the host response of $\mathrm{PBC}$ during the first 2 weeks of the infection.

\section{Results}

The response in peripheral blood of patients with ulceroglandular tularemia was analyzed using Affymetrix U133A microarrays comprising 14500 genes. Samples were categorized in relation to the suspected day of acquiring the infection. Samples were obtained from each of seven individuals at five time points during the first 2 weeks of infection and a convalescent sample approximately 3 months later. RNA was prepared from each sample and analyzed on separate arrays. A pool of RNA from eight healthy volunteers was analyzed and used as control. Data were normalized using quantile normalization $^{7}$ and analyzed with the B statistic. ${ }^{8}$ The results were filtered to include only those spots for which the ratio was at least twofold up- or downregulated compared to the control pool and with a B statistic above zero. Overall, 265 differentially expressed genes were detected in samples collected during the 2-week period, and 95 of these were regulated at more than one time point but not in the convalescent samples. In the convalescent samples, 31 of the genes were differentially expressed. This number was lower than in samples obtained at any time point during the initial 2-week period (Table 1). We categorized differentially expressed genes with clear identities and characterized functions; in total 220 genes (Figure 1). The largest categories were nucleic acid binding (45 genes), transcription factors (21 genes), oxidoreductase activity (19 genes), receptors (18 genes), hydrolase activity (15 genes) and defense/immunity (11 genes).

Of the 265 differentially regulated genes, 39 were selected for confirmatory analysis as they represented well-characterized genes. The differential expression of 36 of these genes was confirmed by quantitative polymerase chain reaction (q-PCR) (Table 2 ). Although the magnitudes of the differential expression varied to some extent between the microarray and Q-PCR analyses, overall there was a significant agreement, $R^{2}=0.590$ (Figure 2).

An upregulation at all five time points was found for the mitochondrial protein ATP synthase (ATP5C1). The protein has been identified in the phagosomal fraction of F. tularensis-infected macrophages ${ }^{9}$ and appears to be a constitutive component of latex bead-containing phagosomes. ${ }^{10}$ Although its role in anti-bacterial defense is unknown, it is co-regulated with TIR-1, which is important to the defense of Caenorhabditis elegans against bacterial infections. ${ }^{11}$

An activator of caspase-mediated apoptosis, TNFSF10 (TRAIL), is a cytokine that belongs to the tumor necrosis factor (TNF) ligand family. It was strongly upregulated at the first time point. This protein binds to several members of the TNF receptor superfamily. ${ }^{12}$ It plays an important role in Helicobacter pylori-mediated apoptosis. ${ }^{13}$ Caspase 1 , which was upregulated at the 2-3 and 10-11 days time points, is a member of the cysteineaspartic acid protease family. Sequential activation of caspases plays a central role in the execution phase of cell apoptosis and caspase 1 also cleaves the proinflammatory mediator interleukin (IL)-1. ${ }^{14}$ Modulation of the gene expression has been found to be essential for the pathogenicity of Salmonella infection. ${ }^{15}$ Serum and glucocorticoid-inducible kinase (SGK) is a serine/threonine protein kinase shown to be important in activating

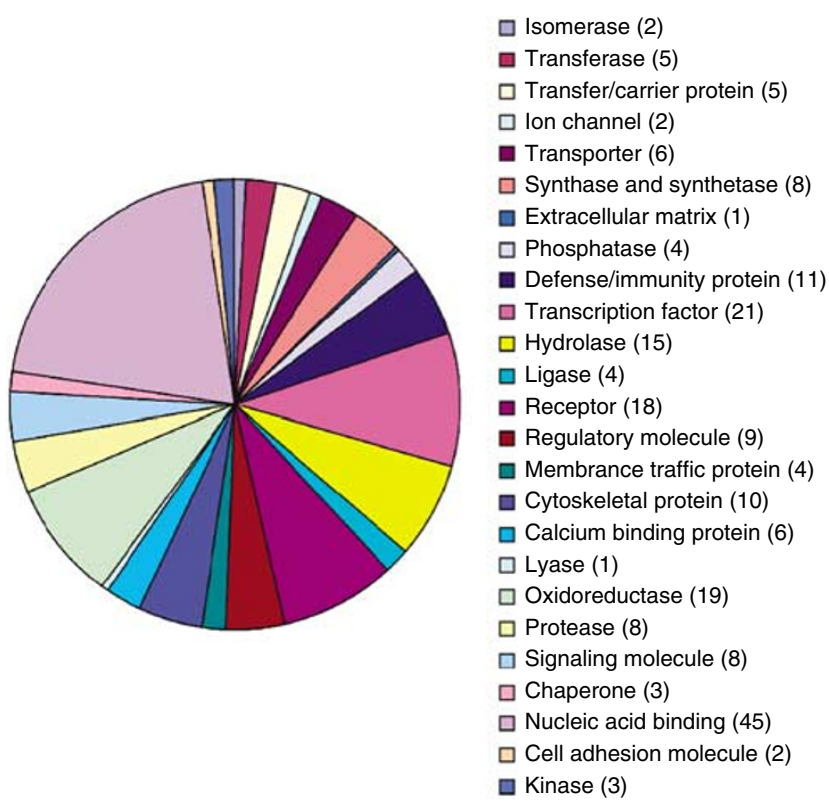

Figure 1 Genes $(n=220)$ identified by microarray analysis as differentially expressed in PBCs of seven tularemia patients compared to eight healthy volunteers were categorized according to gene function as defined in the Gene Ontology database. The remaining 45 differentially regulated genes had no characterized function. Numbers indicate the number of genes in each class.

Table 1 Genes differentially regulated in seven patients with verified F. tularensis infection

\begin{tabular}{lcccrrr}
\hline & Days 2-3 & Days 6-7 & Days 8-9 & Days 10-11 & Day 13 & Convalescent \\
\hline Upregulated & $87^{\mathrm{a}}$ & 69 & 70 & 119 & 91 & 24 \\
Downregulated & 14 & 17 & 7 & 3 & 3 & 3 \\
Total & 101 & 86 & 77 & 122 & 94 & 31 \\
\hline
\end{tabular}

${ }^{a}$ Numbers indicate the number of genes in peripheral blood of patients with ulceroglandular tularemia with a ratio at least twofold up- or downregulated compared to a control pool from eight healthy volunteers and with a B value above zero. Ninety-five genes were regulated at more than one time point but not in the convalescent samples. A total of 14500 genes were analyzed. 
Table 2 Validation by Q-PCR of selected genes identified as differentially regulated by MA ${ }^{\text {a }}$

\begin{tabular}{|c|c|c|c|c|c|c|c|c|c|c|c|c|c|c|}
\hline \multirow[b]{2}{*}{ UniGene ID } & \multirow[b]{2}{*}{ Gene symbol } & \multicolumn{2}{|c|}{ Days 2-3 } & \multicolumn{2}{|c|}{ Days 6-7 } & \multicolumn{2}{|c|}{ Days 8-9 } & \multicolumn{2}{|c|}{ Days 10-11 } & \multicolumn{2}{|c|}{ Day 13} & \multicolumn{2}{|c|}{ Convalescent } & \multirow{2}{*}{$\begin{array}{l}\text { Log. Reg. } \\
\text { Pseudo-R }\end{array}$} \\
\hline & & $M A$ & $Q-P C R$ & $M A$ & $Q-P C R$ & $M A$ & $Q-P C R$ & $M A$ & $Q-P C R$ & $M A$ & $Q-P C R$ & $M A$ & $Q-P C R$ & \\
\hline Hs.271135 & ATP5C1 & 1.11 & 1.15 & 1.33 & 1.35 & 1.25 & 1.43 & 1.55 & 1.93 & 1.18 & 1.48 & 0.63 & 0.77 & 0.220 \\
\hline Hs.377484 & BAG1 & -1.10 & -0.67 & -1.47 & -1.17 & -0.59 & -0.08 & -0.80 & 0.53 & -0.47 & 0.57 & -0.52 & -0.38 & 0.175 \\
\hline Hs.515785 & BLVRB & -0.38 & 0.50 & -1.29 & -0.38 & -0.51 & 0.53 & -0.67 & 0.77 & -0.37 & 0.67 & -0.13 & 0.18 & 0.038 \\
\hline Hs. 2490 & CASP1 & 1.10 & 1.22 & 0.70 & -0.08 & 0.34 & 0.33 & 0.69 & 1.10 & 0.39 & 0.58 & 0.17 & 0.22 & 1.000 \\
\hline Hs.370036 & CCR7 & -1.24 & -1.14 & -0.66 & -0.97 & -0.89 & -0.69 & -0.80 & -0.79 & -0.83 & -0.67 & -0.66 & -0.51 & 0.511 \\
\hline Hs.3003 & CD3E & -1.12 & -0.86 & -0.38 & -0.36 & 0.05 & 0.34 & 0.09 & 0.26 & 0.13 & 0.43 & 0.15 & 0.25 & 1.000 \\
\hline Hs.79630 & CD79A & -1.05 & -1.46 & -1.22 & -2.15 & -0.95 & -1.84 & -1.32 & -1.29 & -0.68 & -0.23 & -0.49 & -0.66 & 0.228 \\
\hline Hs. 85258 & CD8A & -1.00 & -1.13 & -0.45 & -0.16 & -0.44 & 0.02 & -0.35 & -0.07 & -0.39 & -0.58 & -0.42 & -0.10 & 0.556 \\
\hline Hs.70312 & COX7A2 & 1.26 & 2.19 & 1.53 & 2.17 & 1.66 & 2.73 & 1.99 & 3.24 & 1.56 & 3.14 & 0.97 & 1.85 & 0.124 \\
\hline Hs.458485 & G1P2 & 1.78 & 2.49 & 0.90 & 0.75 & 0.88 & 1.77 & 1.01 & 1.98 & 0.74 & 2.22 & 0.28 & 1.29 & 0.638 \\
\hline Hs.62661 & GBP1 & 3.10 & 3.06 & 1.47 & 0.73 & 1.08 & 0.96 & 1.35 & 2.20 & 0.77 & 0.49 & 0.61 & -0.01 & 1.000 \\
\hline Hs. 86724 & GCH1 & 1.90 & 1.50 & 0.99 & 0.23 & 0.31 & 0.17 & 0.97 & 0.91 & 0.71 & -0.26 & 0.64 & -0.45 & 1.000 \\
\hline Hs.28988 & GLRX & 1.86 & 2.36 & 1.32 & 1.48 & 1.11 & 1.79 & 1.41 & 2.50 & 0.82 & 1.71 & 0.39 & 1.15 & 1.000 \\
\hline Hs.105806 & GNLY & -1.11 & -2.67 & 0.34 & -1.50 & 0.87 & -0.48 & 0.73 & -0.32 & 1.04 & -0.25 & 0.64 & -1.00 & 0.882 \\
\hline Hs.1051 & GZMB & -0.14 & 0.37 & 1.19 & 0.98 & 1.26 & 1.27 & 1.26 & 1.35 & 1.47 & 1.78 & 0.39 & 0.28 & 0.080 \\
\hline Hs. 380250 & IFI16 & 1.83 & 0.83 & 0.82 & 0.10 & 1.20 & 0.55 & 1.12 & 1.02 & 0.78 & 0.48 & 0.78 & 0.25 & 0.571 \\
\hline Hs.47338 & IFIT3 & 1.71 & 2.19 & 0.55 & 0.41 & 0.36 & 1.02 & 0.76 & 1.91 & 10.14 & 0.76 & 0.16 & 0.28 & 0.660 \\
\hline Hs.418291 & IL10RB & 1.16 & -0.39 & 0.32 & -1.06 & 0.01 & -0.82 & 0.27 & -0.42 & 0.24 & 0.32 & 0.31 & -0.61 & 0.698 \\
\hline Hs.474787 & IL2RB & -1.06 & -0.98 & -0.64 & -0.39 & -0.54 & 0.09 & -0.55 & 0.21 & -0.27 & -0.16 & -0.23 & 0.09 & 1.000 \\
\hline Hs.69328 & LY96 & 2.17 & 2.38 & 1.27 & 0.98 & 1.13 & 1.40 & 1.71 & 2.93 & 1.18 & 1.74 & 1.07 & 1.35 & 0.511 \\
\hline Hs.461420 & MSRB2 & 1.29 & 1.32 & 0.44 & -0.07 & 0.46 & 0.52 & 0.36 & 0.88 & 0.30 & 0.43 & 0.09 & 0.11 & 1.000 \\
\hline Hs.466871 & PLAUR & 1.43 & 1.11 & 0.80 & -0.13 & 0.37 & 0.12 & 0.43 & 0.26 & -0.22 & -0.06 & 0.05 & -0.10 & 0.784 \\
\hline Hs.434081 & PSME2 & 1.62 & 1.84 & 1.04 & 0.88 & 0.74 & 1.03 & 0.88 & 1.62 & 0.74 & 1.57 & 0.23 & 0.41 & 1.000 \\
\hline Hs. 374588 & RPL17 & 0.63 & 1.91 & 1.04 & 1.73 & 1.11 & 1.81 & 1.79 & 3.13 & 1.18 & 2.21 & 0.95 & 1.75 & 0.013 \\
\hline Hs.546290 & RPS18 & -0.08 & 0.37 & 0.65 & 0.87 & 0.71 & 1.21 & 1.16 & 1.71 & 0.77 & 1.84 & 0.51 & 1.20 & 0.261 \\
\hline Hs.416073 & S100A8 & 1.50 & 2.77 & 1.10 & 2.07 & 1.05 & 2.20 & 1.11 & 2.86 & 0.53 & 2.05 & 0.35 & 1.32 & 0.728 \\
\hline Hs.546296 & SECTM1 & 1.73 & 1.39 & 0.35 & -0.52 & 0.13 & -0.15 & 0.06 & 0.89 & -0.24 & -0.70 & -0.07 & -0.31 & 1.000 \\
\hline Hs. 296323 & SGK & -1.31 & -1.34 & -1.01 & -1.57 & -1.22 & -0.91 & -0.96 & -1.09 & -0.67 & -1.02 & -0.26 & -0.42 & 0.641 \\
\hline Hs.443914 & SOD1 & 0.49 & 0.01 & 0.75 & 0.22 & 0.88 & 0.42 & 0.77 & 0.91 & 1.06 & 1.17 & 0.33 & 0.45 & 0.080 \\
\hline Hs.487046 & SOD2 & 1.23 & 0.70 & 0.58 & -1.07 & 0.03 & -0.47 & 0.25 & 0.07 & 0.39 & -0.85 & 0.44 & -0.38 & 0.743 \\
\hline Hs.470943 & STAT1 & 2.39 & 2.23 & 1.31 & 0.19 & 0.89 & 0.74 & 0.92 & 1.23 & 0.54 & 0.53 & 0.50 & 0.25 & 1.000 \\
\hline Hs.352018 & TAP1 & 1.50 & 1.45 & 0.92 & 0.14 & 0.17 & 0.57 & 0.51 & 1.02 & 0.09 & 0.52 & -0.04 & -0.01 & 1.000 \\
\hline Hs. 437322 & TNFAIP6 & 2.26 & 3.02 & 1.28 & 0.77 & 0.87 & 0.82 & 1.18 & 2.31 & 0.63 & 0.83 & 0.48 & 0.44 & 1.000 \\
\hline Hs. 478275 & TNFSF10 & 2.22 & 1.63 & 0.99 & 0.32 & 0.68 & 0.56 & 1.23 & 1.43 & 0.48 & 0.21 & 0.58 & 0.27 & 1.000 \\
\hline Hs.58831 & TOSO & -1.12 & -1.48 & -0.56 & -1.48 & -0.60 & -0.85 & -0.45 & -1.17 & -0.33 & -0.91 & -0.28 & -0.47 & 0.691 \\
\hline Hs.534032 & TRGV9 & -1.03 & -1.01 & 0.74 & 1.12 & 1.50 & 2.49 & 1.67 & 2.93 & 1.68 & 2.39 & 1.46 & 2.08 & 0.890 \\
\hline
\end{tabular}

Abbreviations: MA, microarray analysis; Q-PCR, quantitative polymerase chain reaction.

${ }^{a}$ Genes identified as differentially expressed by microarray analysis were chosen for validation. cDNA from seven patients were pooled and analyzed by Q-PCR. Of 39 genes, 36 genes were verified as differentially expressed. Values represent $\log _{2}$ ratios between the indicated sample and that of healthy volunteers. Microarray (MA) values represent mean $\log _{2}$ ratios from 6 to 7 biological replicates. Q-PCR values represent mean $\log _{2}$ ratios of three technical replicates. MA results with B values $>2$ and Q-PCR results with $P<0.05$ are considered significant and are marked in bold.

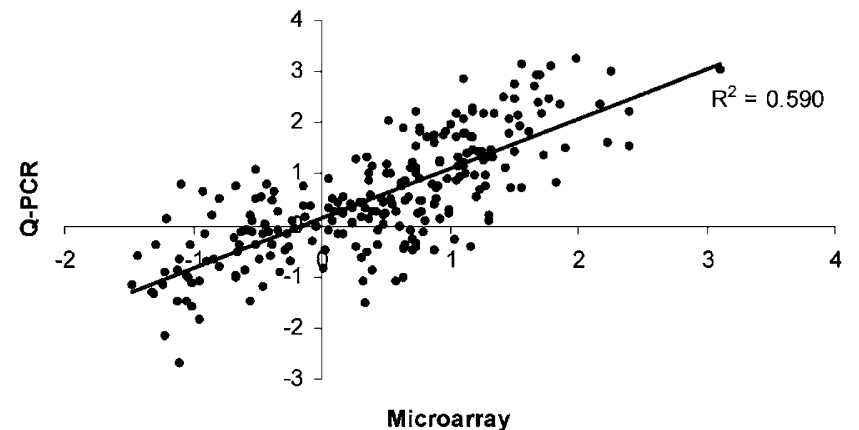

Figure 2 Comparison of the ratios obtained from microarray analysis and Q-PCR analysis for 39 genes identified by microarray to be differentially expressed in seven tularemia patients compared to eight healthy volunteers. There was a significant correlation between the microarray analysis and Q-PCR data $\left(R^{2}=0.590\right)$.

certain ion channels and it plays a crucial role in promoting cell survival via a nuclear factor (kappa)B (NF- $\kappa$ B)-dependent mechanism. ${ }^{16}$ It was found to be persistently downregulated. A similar downregulation of TOSO was observed. It is an inhibitor of apoptosis mediated by members of the TNF receptor family and Fas. ${ }^{17}$ Altogether, the evidence indicates that a proapoptotic response occurred during the course of the disease as there was upregulation of proapoptotic genes and downregulation of antiapoptotic genes.

Interaction of chemokine receptor CCR7 on lymphocytes with its ligands CCL19 and CCL21 expressed on high endothelial venules is essential for effective migration of $\mathrm{T}$ and $\mathrm{B}$ cells into secondary lymphoid organs. ${ }^{18}$ The CCR7 gene was downregulated at the 2-3 days time point. Moreover, at the same time point, several other genes important for executing immune functions were downregulated, for example, the CD3 epsilon chain, the IL-2B receptor, the T-cell receptor CD8A, a component of the B-cell antigen receptor, CD79A and the T-cell effector granulolysin, GNLY. IL-2 is required for expansion of T cells and CD3 is essential for eliciting T-cell-effector function. Also in tuberculosis patients, a downregulation of CD3 has been observed. ${ }^{19}$ At the same, first time point, 
TSG-6 (TNFAIP6) was upregulated. The protein possesses potent anti-inflammatory properties, but the precise mechanism(s) is elusive and, therefore, whether its upregulation was in any way related to the aforementioned downregulation of a number of immune effector genes is unknown. ${ }^{20}$

Thus, it is possible that certain intracellular pathogens attempt to subvert innate and acquired immune mechanisms by specifically downregulating genes critically required for generation of an effective anti-bacterial response. By contrast, certain genes contributing to the development of an immune response were upregulated. For example, the calcium binding protein S100 CP-10 (S100A8) was persistently upregulated. It is a potent chemotactic factor for murine and human myeloid cells in vivo and in vitro and is regulated by proinflammatory stimuli. It has been proposed that it plays a role in recruitment of leukocytes into tissues in response to Gram-negative bacterial infection. ${ }^{21}$ Also MD-2 (LY96) was persistently upregulated. It is a crucial molecule interacting with Toll-like receptor (TLR)4, which enhances the binding of enterobacterial lipopolysaccharide (LPS) to TLR4-expressing cells. In fact, it has been suggested that binding of MD-2 to LPS is necessary for the interaction of the latter with TLR4. ${ }^{22}$ Despite its key role in TLR-mediated signaling, little is known about its regulation during infection and therefore it is difficult to make any conclusion about the relevance of its persistent upregulation.

A number of interferon (IFN)-regulated genes or genes affecting IFN- $\gamma$ were found to be upregulated during one or several phases of infection. These include G1P2, guanylate-binding protein (GBP)-1, GCH1, IFI16, IFIT3, signal transducers and activators of transcription (STAT)1, granzyme B (GZMB) and transport of antigenic peptides (TAP)-1. G1P2 is a ubiquitin homologue and a cytokine that induces production of IFN- $\gamma$ and augments natural killer cell function. GBP-1 is strongly induced by IL-1 and IFN- $\gamma$. Both are important for the control of several viral infections and are crucial mediators of IFNmediated immune responses. ${ }^{23} \mathrm{GCH} 1$ is the first and rate-limiting enzyme in tetrahydrobiopterin (BH4) biosynthesis. ${ }^{24}$ Induction of GCH1 and nitric oxide synthase (NOS) has been suggested to represent two arms of a common pathway required for immunostimulantevoked NO synthesis, and the major function of $\mathrm{BH} 4$ may be to support NOS. ${ }^{25}$ STATs are activated in response to all cytokines that utilize cytokine receptor superfamily members. This receptor family transduces signals for approximately 30 cytokines having diverse biological functions. The only non-redundant role of STAT1 appears to be to regulate a set of genes that collectively confer innate immunity. ${ }^{26}$ It was upregulated in samples from the 2 to 3 days time point but also downregulated in samples from the day 13 time point and in convalescent samples. GZMB is present in the granules of cytolytic lymphocytes and is a crucial mediator of cell-mediated target cell death via the granule-mediated pathway, thereby an important effector for protection against many types of infections. The lytic ability of cytotoxic T cells is induced by IFN- $\gamma$. We observed a persistent upregulation of GZMB from the days 6 to 7 time point until day 13. The TAP from the immunoproteasome is mediated via TAP proteins. Its expression is under control of IFN- $\gamma$ and STAT1. ${ }^{27}$
Another protein controlling the proteasome is BAG-1 (Bcl-2-associated athanogene), which is a multifaceted protein involved in the modulation of a large variety of cellular processes. It was found to be downregulated at the first two time points. One reason for its multifunctional roles appears to be its interaction with molecular chaperones of the Hsp70 family. Thus, it possibly coordinates chaperone and degradation pathways.

Glutaredoxin (GLRX) is a glutathione (GSH)-dependent hydrogen donor for ribonucleotide reductase and also catalyzes glutathione-disulfide oxidoreduction reactions in the presence of nicotinamide adenine dinucleotide phosphate (reduced form) and glutathione reductase. It appears to be a host defense mechanism to protect macrophages against oxidative damages. ${ }^{28}$ GLRX was strongly and persistently activated. Also methionine sulfoxide reductase B (MSRB)2 appears to play an important role in repairing oxidative damage to proteins that may occur during infection and it was also upregulated, most prominently at the first time point. Also other genes encoding enzymes with anti-oxidative functions were found to be transiently upregulated. The mitochondrial antioxidant manganese superoxide dismutase-2 (Sod2) and the $\mathrm{CuZn}$ superoxide dismutase (Sod1) genes were upregulated at the days 2-3 and day 13 time points, respectively. In contrast, one gene encoding an enzyme, biliverdin reductase (BLVRB), possibly involved in an anti-oxidative response was transiently downregulated at the days 6-7 time point. It is a kinase that catalyzes reduction of the heme oxygenase product, biliverdin, to bilirubin. It has been suggested to play a role in the signaling cascade of the transcription factor AP-1 necessary for heme oxygenase oxidative stress response.

The binding of urokinase-type plasminogen activator to its receptor (PLAUR) affects a variety of functions such as vascular homeostasis, inflammation and tissue repair. It is also a predictor of HIV disease progression. We observed an upregulation at the first time point. Upregulation of host plasminogen activator receptors has been observed during other bacterial infections, for example, with Staphylococcus aureus and Borrelia burgdorferi. ${ }^{29}$

A strong and persistent upregulation of the cytochrome $c$ oxidase VIIa polypeptide 2 (COX7A2) was found. COX is the terminal component of the mitochondrial respiratory chain, which catalyzes the electron transfer from reduced cytochrome $c$ to oxygen. This nuclear-encoded subunit may function in the regulation and assembly of the COX complex. Although the reason for this upregulation is not clear, COX is upregulated during apoptosis through the mitochondrial pathway induced by Streptococcus pyogenes. ${ }^{30}$

A large number of ribosomal genes appeared to be coregulated and were found to be persistently upregulated during infection, as exemplified by RPL17. This may be an indication of a need for increased protein synthesis to prepare the cell to fight the infection.

TRGV9 is a gene expressed by a subset of $\gamma \delta$ T cells, socalled $\mathrm{V} \gamma 9 \mathrm{~V} \delta 2 \mathrm{~T}$ cells. We have previously demonstrated that tularemia patients exhibit a long-lasting and very prominent increase of this cell type. ${ }^{31}$ It appears as if they play a part in the early immune response to infection, perhaps by initially secreting high levels of cytokines 
Table 3 Q-PCR analysis of cytokine mRNA levels in PBCs of tularemia patients ${ }^{a}$

\begin{tabular}{|c|c|c|c|c|c|c|}
\hline Gene & Days 2-3 & Days 6-7 & Days 8-9 & Days 10-11 & Day 13 & Convalescent \\
\hline IFN- $\gamma$ & 0.36 & 1.28 & 1.04 & 1.87 & 0.93 & 0.31 \\
\hline TNF- $\alpha$ & 0.11 & -0.65 & -0.43 & -0.36 & -0.76 & -0.13 \\
\hline IL-1 $\alpha$ & ND & ND & ND & ND & ND & ND \\
\hline IL- $1 \beta$ & 0.56 & -1.07 & -0.65 & 0.31 & -1.55 & -0.95 \\
\hline IL-2 & -0.45 & -0.38 & 0.09 & 0.66 & -0.04 & 0.35 \\
\hline IL-4 & 0.46 & -0.14 & 0.03 & 0.89 & 0.45 & 0.40 \\
\hline IL-5 & ND & ND & ND & ND & ND & ND \\
\hline IL-8 & -0.66 & -1.55 & -0.42 & -0.24 & -0.71 & -0.55 \\
\hline IL-10 & 0.72 & -0.55 & -0.37 & 0.18 & -0.09 & -1.19 \\
\hline IL-12p35 & 0.04 & -0.38 & 0.77 & 1.23 & 0.63 & -0.46 \\
\hline IL-12p40 & ND & ND & ND & ND & ND & ND \\
\hline IL-15 & 1.30 & 0.56 & 0.46 & 1.37 & 0.19 & 0.30 \\
\hline
\end{tabular}

Abbreviations: IFN, interferon; IL, interleukin; mRNA, messenger RNA; ND, not detected; PBC, peripheral blood cell; Q-PCR, quantitative polymerase chain reaction; $\mathrm{TNF}$, tumor necrosis factor.

${ }^{\mathrm{a}} \mathrm{CDNA}$ from seven patients was pooled and the expression of 12 cytokines was analyzed by Q-PCR. Values represent mean log ${ }_{2}$ ratios $(n=2)$ between the indicated sample and that of healthy volunteers.

such as IFN- $\gamma$ and $\mathrm{TNF}-\alpha$, and subsequently downmodulating this secretion. ${ }^{32}$ Notable, RNA levels were much higher in the patient convalescent samples obtained 3 months after recovery than in the pooled control RNA sample. This agrees with the previous demonstration that markedly higher levels of $\mathrm{V} \gamma 9 \mathrm{~V} \delta 2 \mathrm{~T}$ cells persisted in peripheral blood at least 18 months after tularemia. ${ }^{32}$

We performed Q-PCR analysis of cytokine gene expressions (Table 3). We found a significant change in mRNA levels for IFN- $\gamma$, TNF- $\alpha$, IL- $1 \beta$ and IL-15. Owing to their transcriptional regulation, they were chosen for analysis of serum levels. An increase of IFN- $\gamma$ levels was observed at the 2-3 days time point but, besides an individual sample, not thereafter (Figure 4). Levels of TNF- $\alpha$, IL- $1 \beta$ and IL- 15 were below the detection limits in all samples (data not shown).

To search for co-regulation of genes with similar function, we subjected all genes that were differentially regulated to K-means cluster analysis (Figure 3). The stability of the clusters was estimated using a parametric resampling procedure as described by Zhang and Zhao. ${ }^{33}$ The stability index indicates the percentage of resampled clusters that included more than $80 \%$ of the genes in the original cluster. Thus, a higher stability index indicates a more stable cluster. The percentages are presented in Figure 3. These clusters were tested against the NCBI human gene list to search for over- or underrepresented functional categories. Clusters 1 and 9 had an overrepresentation of genes involved in defense and immunity. The genes in cluster 1 were downregulated at all time points. Cluster 9 consisted of genes upregulated at the days 2-3 time point. Clusters 2, 3 and 5 consisted of genes upregulated at all time points and showed an overrepresentation of ribosomal genes, genes coding for nucleic acid-binding proteins and calmodulinrelated proteins, respectively. Cluster 7 had an overrepresentation of hydrolases and oxidoreductases.

A core of some 500 genes has been suggested to constitute a general 'alarm signal' for infection and the differential regulation of these genes has been identified to occur in macrophages, dendritic cells (DC) and peripheral blood mononuclear cells (PBMC) during many different forms of infection. ${ }^{34}$ On the Affymetrix
U133A arrays, 260 of the 511 core-cluster genes were present and transcripts from 104 'alarm-signal' genes were detected in our experiments. Only 16 were found to be differentially expressed (Table 4), 14 of which were upregulated, whereas BAG-1 was found to be downregulated at two time points and Pim-1 downregulated at days 6-7.

We performed logistic regression analysis to identify those genes that best classify tularemia patients at the days 2-3 time point (before start of treatment) compared to convalescent samples. Ranking was based on pseudo$R^{2}$ values ( $R^{2}$ values for logistic regression) (Table 2) calculated as described by Nagelkerke. ${ }^{35}$ The probabilities for the 19 genes that gave best separation between tularemia samples and convalescent samples are shown in Figure 5. Of these, 13 genes gave a complete separation (pseudo $R^{2}=1$ ) and six of these were among the aforementioned 'alarm-signal' genes. The remaining, which can be considered as putative early diagnostic markers of tularemia, were STAT1, SECTM1, TNFAIP6, TNFSF10, CD3E, MSRB2 and IL2RB.

\section{Discussion}

F. tularensis is classified as a category A agent, thus being a likely bioterrorism agent and therefore the focus of an intense current research effort. Still, very little is known about the pathogenesis of tularemia and virulence mechanisms of F. tularensis. One way to learn about the molecular interactions during an infection is to analyze the host response. To this end, we carried out a systematic analysis of the adaptation of PBC during the course of tularemia. The results provide a picture of pathogenetic mechanisms active during the infection and identify issues that require future investigations.

In a previous study, we characterized the transcriptional response in mice to an aerosol infection with F. tularensis subsp tularensis (type A). ${ }^{36}$ A prominent finding was that many of the induced genes were known to be dependent on TNF- $\alpha$ or IFN- $\gamma$ for their regulation and marked increases of the cytokines in bronchoalveolar lavage samples were noted by day 4 of infection. Despite this immune response of a Th1 type, the type A 

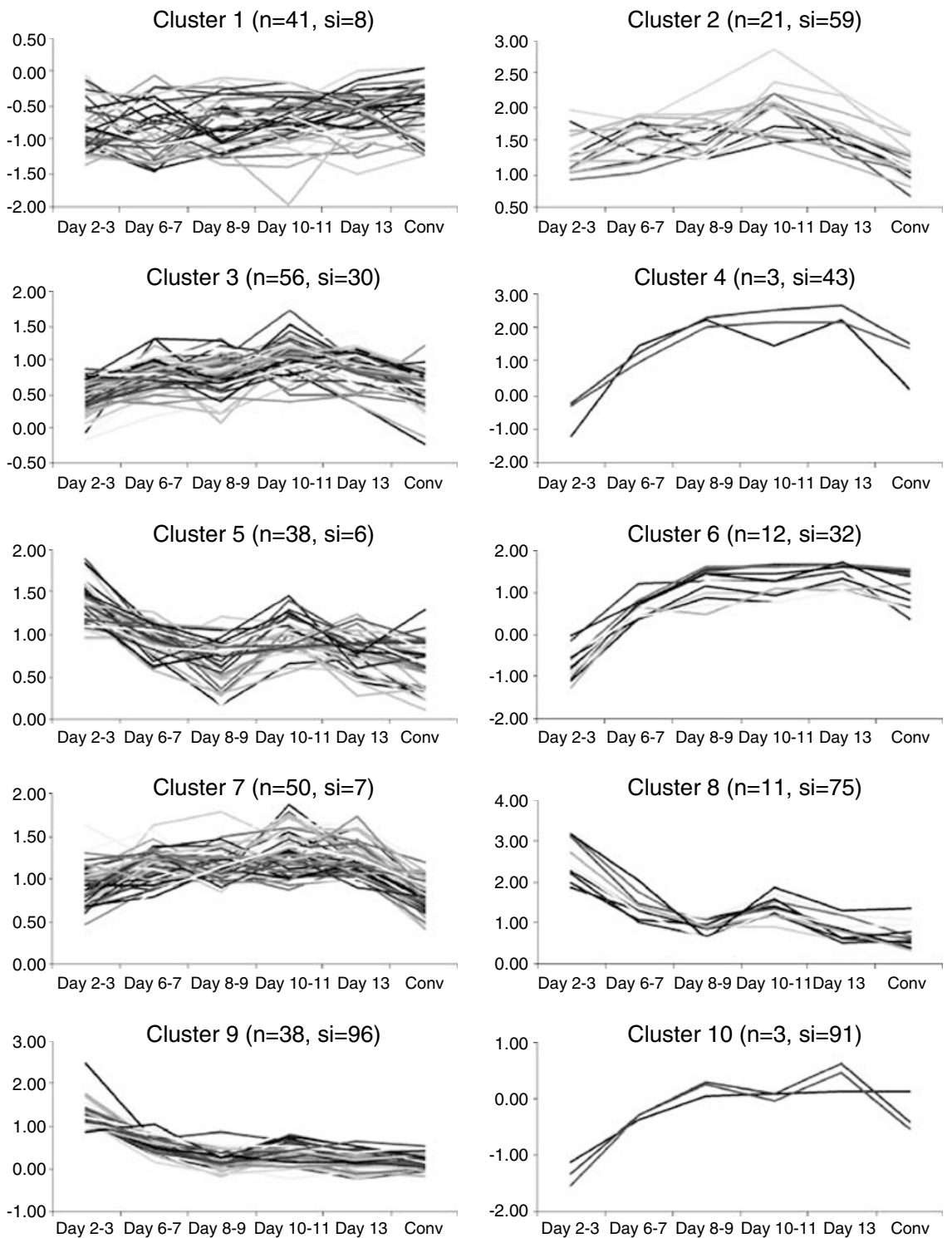

Figure 3 K-means clustering of 273 probesets corresponding to 265 unique genes up- and downregulated in seven tularemia patients compared to eight healthy volunteers. Here, si denotes the stability index; a higher index indicates a more stable cluster.

infection is uniformly lethal in mice. ${ }^{37}$ Our present findings indicate that many of the genes prominently upregulated during the early phase of human tularemia are also dependent on IFN $-\gamma$ for their expression. Previous studies have demonstrated that a protective, cell-mediated immune response develops during the second week of infection. ${ }^{38}$ Despite the early onset of a presumably protective immune response, tularemia may persist in humans for several weeks. Our present findings may help to explain some of these apparently paradoxical findings, as we observed downregulation of a number of factors known to be critically required for effectuating various facets of an immune response, such as CCR7 for recruitment of leukocytes, IL-2RB, CD3 epsilon, CD8 $\alpha$ and GNLY, all required for an optimal T-cell effector function and CD79, a component of the B-cell receptor. Thus, it is possible that specific downregulation of key immune effector genes counteracts the developing protective immune response and allows persistence of the infection. Moreover, serum levels of proinflammatory cytokines were not increased at all (Table 3 and Figure 4) with the exception of IFN- $\gamma$, which showed an initial, transient increase. The findings fit with the view that $F$. tularensis has a stealth strategy to ensure intracellular survival and with the rather inconspicuous clinical manifestations during the disease. ${ }^{39}$ It should be noted that antibiotic therapy was instituted at some time during the first 6 days of disease in all patients. The treatment may have contributed to the minimal inflammatory response thereafter.

Our previous studies of the course of an F. tularensis infection in murine macrophages revealed that the cells are killed by apoptosis within $24-48 \mathrm{~h} .{ }^{40,41}$ The apoptosis is mediated via a pathway similar to the intrinsic pathway with involvement of the mitochondria and subsequent activation of caspase- 9 and caspase- $3 .{ }^{40}$ The present study also identified activation of apoptotic mechanisms during ulceroglandular tularemia, although these may not directly mimic the pathways involved in murine macrophages. We observed upregulation of 
Table 4 Genes belonging to the 'alarm-signal' cluster that were found to be differentially expressed in PBCs of tularemia patients

\begin{tabular}{|c|c|c|c|c|c|c|c|}
\hline \multirow[t]{2}{*}{ Gene annotation } & \multirow[t]{2}{*}{ Gene symbol } & \multicolumn{6}{|c|}{ Time point } \\
\hline & & $2-3$ & $6-7$ & $8-9$ & $10-11$ & 13 & Convalescent \\
\hline BCL2-associated athanogene & BAG1 & $-1.10^{\mathrm{b}}$ & -1.47 & -0.59 & -0.80 & -0.47 & -0.52 \\
\hline Caspase 1 & CASP1 & 1.10 & 0.70 & 0.34 & 0.69 & 0.39 & 0.17 \\
\hline Chemokine (C-C motif) ligand 4 & CCL4 & -0.16 & 0.17 & 0.37 & 0.95 & 1.03 & 0.60 \\
\hline CD59 antigen p18-20 & CD59 & 1.01 & 0.70 & 0.87 & 0.65 & 0.43 & 0.11 \\
\hline Fc fragment of $\operatorname{IgE}$ receptor gamma & FCER1G & 1.14 & 0.53 & 0.08 & 0.03 & 0.10 & 0.07 \\
\hline Interferon alpha-inducible protein & G1P2 & 1.78 & 0.90 & 0.88 & 1.01 & 0.74 & 0.28 \\
\hline Guanylate binding protein 1 & GBP1 & 3.10 & 1.47 & 1.08 & 1.35 & 0.77 & 0.61 \\
\hline GTP cyclohydrolase 1 & GCH1 & 1.90 & 0.99 & 0.31 & 0.97 & 0.71 & 0.64 \\
\hline Glutaredoxin (thioltransferase) & GLRX & 1.86 & 1.32 & 1.11 & 1.41 & 0.82 & 0.39 \\
\hline Interferon induced transmembrane protein 1 & IFITM1 & 1.07 & 0.48 & 0.10 & 0.41 & 0.12 & 0.22 \\
\hline Karyopherin alpha 2 & KPNA2 & 1.08 & 1.46 & 1.09 & 1.02 & 1.38 & 0.55 \\
\hline N-myc (and STAT) interactor & NMI & 1.18 & 0.84 & 0.29 & 0.85 & 0.52 & 0.15 \\
\hline pim-1 oncogene & PIM1 & -0.81 & -1.09 & -0.87 & -0.84 & -0.54 & -0.49 \\
\hline Proteasome activator subunit 2 & PSME2 & 1.62 & 1.04 & 0.74 & 0.88 & 0.74 & 0.23 \\
\hline Spermidine/spermine N1 & SAT & 1.42 & 1.04 & 0.51 & 0.91 & 0.77 & 0.61 \\
\hline Transporter 1 & TAP1 & 1.50 & 0.92 & 0.17 & 0.51 & 0.09 & -0.04 \\
\hline
\end{tabular}

a'Genes identified by microarray analysis as differentially expressed in seven tularemia patients that belong to the 'alarm signal' cluster identified by Jenner and Young. ${ }^{34}$

${ }^{b}$ Values represent mean $\log _{2}$ ratios between the indicated sample and that of healthy volunteers. Results with B values $>2$ are considered significant and are marked in bold.

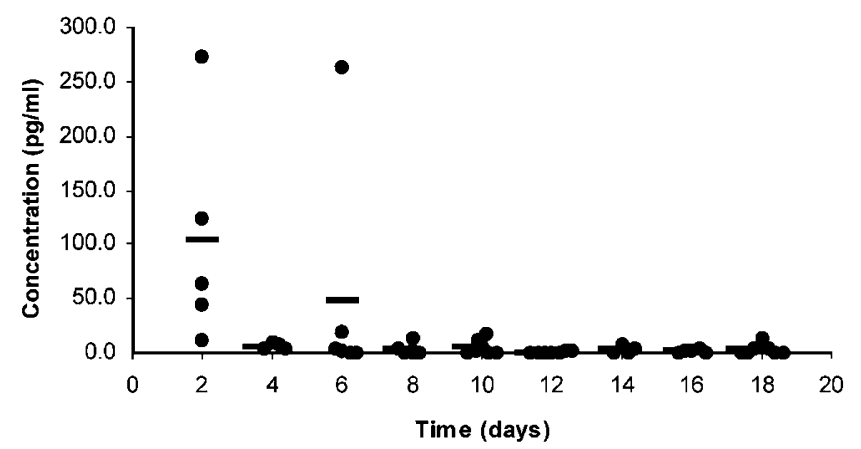

Figure 4 IFN- $\gamma$ levels in serum from tularemia patients during the first 3 weeks after the onset of disease. Each dot on the graph represents the result from an individual patient at that time point ( $n=4-7$ for each time point).

a factor, TNFSF10, known to control other forms of infection-induced apoptosis. Moreover, caspase 1 was upregulated at several time points. SGK, identified as a factor that promotes cell survival, was downregulated together with TOSO, an inhibitor of certain forms of apoptosis. ${ }^{17}$ The overall picture is that of a proapoptotic response. Whether this benefits the pathogen or the host is unclear, but in the murine model, it was suggested that the macrophage-mediated apoptosis favors F. tularensis, as it allows the bacterium to escape by a silent mechanism from heavily infected cells deplete of nutrients and infect neighboring cells replete of nutrients. ${ }^{39}$ The apoptotic nature of host cell death also fits with the general stealth-like nature of tularemia.

Investigations have been performed to describe the transcriptional regulation during various phases of other human or experimental animal model diseases such as smallpox or sepsis. In a murine model of sepsis, $4.8 \%$ of the investigated genes were differentially regulated but only $0.3 \%$ of these discriminated between Gram-negative and Gram-positive sepsis. ${ }^{2}$ In a macaque model of smallpox, generalized responses representing IFN-regulation genes, cell proliferation genes and immunoglobulin genes were observed. ${ }^{1}$ However, also a lack of proinflammatory response involving TNF- $\alpha-$ and NF- $\kappa \mathrm{B}-$ dependent genes was noted, suggesting that smallpox may abrogate this response. The findings from such diverse models support the hypothesis that the various forms of sepsis and other infectious diseases share host effector pathways, ${ }^{2}$ but also demonstrate that subversion of specific immune functions may be pathognomonic of certain diseases. This general conclusion is in line with the present findings on tularemia.

Additional support for shared effector pathways during infectious diseases comes from a review by Jenner and Young ${ }^{34}$ analyzing the transcriptional responses to many types of infections in vitro or in vivo. It was demonstrated that despite fundamental differences in the pathogenicity of the diseases, a core cluster of some 500 genes could be identified as co-regulated. As this gene expression pattern appeared to be common in macrophages, DC and PBMC, it was suggested to constitute a general 'alarm signal' for infection, thus maximizing the likelihood of recognition. A high proportion of these genes encode immune response regulators, in particular cytokines and chemokines. Transcription factors represented another group of differentially expressed immune regulators and a third group was IFN-regulated genes. In our experiments, of 104 'alarm-signal' genes that were detected, only 16 were found to be differentially regulated. In agreement with our previous findings, ${ }^{42} \mathrm{~F}$. tularensis induces a very moderate host transcriptional response, a stealth-like behavior favorable for the pathogen as it minimizes the inflammatory response and possibly delays the onset of the acquired immune response. On the other hand, certain facets of this core-cluster are obviously activated during tularemia, such as IFN-regulated genes. 


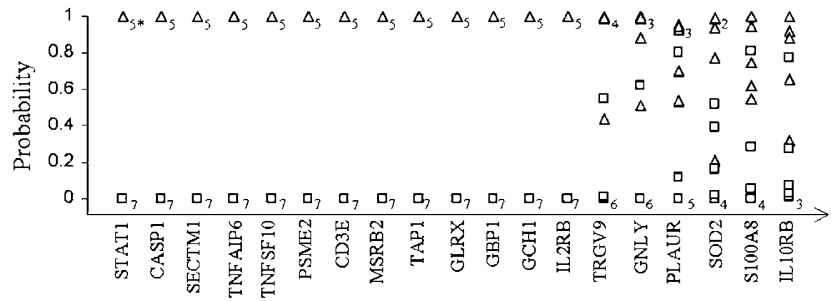

Figure 5 Logistic regression probabilities for 19 genes that best separate days 2-3 tularemia patient samples from convalescent samples taken 3-4 months later. Days 2-3 samples are represented by $\Delta(n=5)$ and convalescent samples by $\square(n=7)$. *Number of samples with equal value.

Logistic regression analysis was used to identify genes that were uniquely regulated during the early phase of tularemia compared to the convalescent samples. We identified 19 genes that gave good separation between these two time points, 13 of which gave a complete separation (Table 2 and Figure 5). Six genes (Caspase-1, PSME2, TAP-1, GLRX, GBP-1 and GCH1) belong to the 'alarm-signal' cluster ${ }^{34}$ and, therefore, are known to be regulated as part of many infectious or inflammatory processes. The remaining seven genes, STAT1, SECTM1, TNFAIP6, TNFSF10, CD3E, MSRB2 and IL2RB, may be used for detection of the early phase of tularemia. As aforementioned, several of the genes encode factors known to be involved in innate immune mechanisms and to this end it can be assumed that they are regulated in response to other infections as well. Although not all of the 19 genes may be uniquely regulated during the early phase of tularemia, it is possible that their combined regulation can be used for diagnostic purposes. In order to obtain a reliable diagnostic tool, samples from a larger patient material need to be analyzed. By identifying such early markers, it may be possible to establish the diagnosis even before a typical clinical presentation appears.

The inclusion of tularemia patients in the study was performed in a prospective manner and a relative low proportion of individuals volunteered. As many of the cases occur during the holiday season in Sweden, this fact may have resulted in a bias giving the inclusion of relatively older individuals in the study. The average age of all tularemia patients from the region during 2003-2004 was 47 years, thus considerably lower than the average age of 61 years of the study group. As it has been demonstrated that age is one important factor affecting the gene transcriptional response, it may explain some of the differential expression patterns found in convalescent samples. Other factors known to affect the patterns of transcriptional responses are the time of day when samples are obtained and gender. ${ }^{43}$ We tried to minimize these confounders by obtaining the samples between 0800 and 1200 hours and by including similar numbers of men and women. Another factor, which to our knowledge never has been studied, is the longevity of infectioninduced modulation of gene transcriptional responses.

Although tularemia generally presents as an acute infection and despite that all patients included in the present and several of our previous studies received early, proper antibiotic treatment, we previously identified a very long-lasting effect on the differential blood leukocyte count. ${ }^{32}$ A prominent increase of circulating
$\mathrm{V} \gamma 9 \mathrm{~V} \delta 2 \mathrm{~T}$ cells occurred between days 8 and 40 and the increase persisted up to 18 months after infection. Considering that these changes are so long-lasting, they may be one likely explanation for some of the transcriptional changes observed in the convalescent samples. The persistent transcriptional regulation warrants further studies to determine whether it is due to an effect of the infection per se or whether other factors, such as age, may have contributed to the bias.

Genes with a ratio of $>2$ and a positive B value were called differentially expressed and were further validated by a Q-PCR analysis. Although the rather stringent selection criteria, some genes were expected to be false positives due to mass significance. In accordance with this, not all of the microarray results were confirmed by the highly sensitive Q-PCR technique $\left(R^{2}=0.590\right)$. Altogether, this comparative analysis demonstrates that a drawback of the microarray analysis is the relative high proportion of false-positive clones when the number of true-positive clones is relatively small.

A mixed cell population, such as PBC studied herein, presents a more complex and comprehensive picture of gene expression during the systemic host responses to infection than does any individual cell type. However, data from mixed cell populations can be more difficult to interpret. Although it may be possible to infer variation in PBC expression to specific cell subsets by correlating the relative numbers of a certain subpopulation and the gene expression patterns, future experiments examining the host response to tularemia in less heterogeneous cell populations will assist in identifying cell type-specific responses. Likewise, examination of different tissues during the course of tularemia will reveal a more detailed picture of the pathogenesis.

Altogether, our results provide a useful foundation for explaining the pathogenesis of ulceroglandular tularemia. Large-scale transcriptional analysis allows for an effective identification of diagnostic and predictive biomarkers and if such markers could be included in rapid, simple assays, they will provide important information to assist in decisions regarding institution of early therapeutic regimens in tularemia patients.

\section{Patients and methods}

Human subjects

In August and September 2003 and 2004, outbreaks occurred in Närke county in central Sweden. All cases visited the Infectious Disease Department of Örebro University Hospital. Seven patients (50-76 years old; mean age of 60.7 years; three females, four males) were included. Treatment with doxycycline was instituted in five individuals after the first hospital visit and in two individuals after the second visit (days 6-7). In all cases, mosquito bites were the indicated source of infection. Samples were collected by the PAX system (Qiagen, Valencia, CA, USA). From each patient, blood samples were drawn at the first visit to the Infectious Disease Department and on 3-4 additional occasions during the next 13 days. An additional sample was drawn 3-4 months later, to be used as a convalescent sample. Essentially all samples were obtained between 0800 and 1200 hours. Samples were also obtained from eight healthy volunteers with no previous history of tularemia 
to be used as a source of control RNA for the microarray analysis. Ethical permission for the sampling was obtained from Örebro University Hospital.

The disease was diagnosed by the demonstration of $F$. tularensis-specific antibodies in blood samples drawn 4-5 weeks after the onset of infection. A tube agglutination test was applied, using a commercially available whole cell antigen (SBL Vaccin, Stockholm, Sweden). A single titer of at least 1/80 or a fourfold increase in titers was regarded as diagnostic. All analyses were performed at the Department of Clinical Microbiology, Örebro University Hospital. Total leukocyte counts varied from 5.9 to $9.4 \times 10^{9} / 1$, with mean lymphocyte counts from 1.1 to $2.7 \times 10^{9} / 1$

\section{Sample processing}

Total RNAs were isolated from the peripheral blood samples using the PAXgene isolation kit (Qiagen). Frozen PAX tubes were thawed at room temperature for $2 \mathrm{~h}$ followed by total RNA isolation as described in the PAX kit handbook, but with the following modifications suggested by Thach et al. ${ }^{44}$ To aid in tight pellet formation, we increased proteinase $\mathrm{K}$ from 40 to $80 \mu \mathrm{l}$ per sample, extending the $55^{\circ} \mathrm{C}$ incubation time from 10 to $30 \mathrm{~min}$, and the centrifugation time to $30 \mathrm{~min}$. The DNase treatment was performed in solution using a DNA-free kit (Ambion Inc., Austin, TX, USA). Prepared RNA from the control samples were pooled and used as a reference. Purified total RNA was stored in ethanol at $-20^{\circ} \mathrm{C}$ until use. RNA samples with a ratio of $A_{260}$ to $A_{280}$ between 2.0 and 2.1 were used for experiments. The isolated RNA was amplified and labeled as described in the GeneChip Expression Analysis Technical Manual, and analyzed using the HG-U133A Genechip microarray (Affymetrix Inc., Santa Clara, CA, USA) according to the manufacturer's recommendations.

\section{Statistical analysis}

Arrays from seven patients with good quality were used for statistical analysis, except for the days 2-3 time point when five samples were obtained and days 8-9 when six samples were obtained. Scaling was carried out for gene expression data in MAS 5.1 software (Affymetrix). All array data were scaled to a target value of 500. Data were then normalized using the quantile normalization method suggested by Bolstad et al. ${ }^{7}$ The normalized data were analyzed using the B-statistic suggested by Lönnstedt et al. ${ }^{8}$ Differentially expressed genes were selected according to ratio and B-statistics.

Logistic regression using the logit transformation was used to model the relationship between the status of the patient and the observed signal of a gene. The pseudo- $R^{2}$ values were calculated as described by Nagelkerke. ${ }^{35}$

K-means clustering was performed using S-Plus 6.1 software (Insightful, Seattle, WA, USA). The clusters were statistically compared to a reference list (NCBI Homo sapiens genes) to look for under- and overrepresented functional categories using the gene expression data analysis tool at Applied Biosystems Panther Classification system, http://www.pantherdb. org/tools/genexAnalysis.jsp.

A resampling procedure similar to the one described by Zhang and $\mathrm{Zhao}^{33}$ was used to assess the reproducibility of the K-means cluster analysis. For each gene $g$ and time point $t$, the average normalized $\log$-ratio $M_{g^{t}}$ and the corresponding standard error $s_{g t}$ were observed.

The cluster analysis on the original data $M$, consisting of the average normalized log-ratios from 265 selected genes, generated 10 original clusters. In our approach, a bootstrap replicate $M^{*}$ was obtained by replacing each element $M_{g t}$ of the original data by a value simulated from a normal distribution $N\left(M_{g t}, s_{g t}\right)$. The cluster analysis applied on the bootstrap replicated data set $M^{*}$ generated 10 new clusters. If more than $80 \%$ of the genes of the original cluster were included in one of the new clusters, then the original cluster was designated as present. In total, 1000 bootstrap replicated data sets were generated. A cluster's stability index indicates the percentage of times the original cluster was designated as present. A high stability index indicated that the original cluster was robust.

\section{Ouantitative real-time PCR}

RNA from the same control and test samples used in the microarray experiments was reverse transcribed into cDNA. Specific primers for selected genes were designed using the Primer3 program available at http://frodo. wi.mit.edu/cgi-bin/primer3/primer3_www.cgi.

Quantitative real-time PCR (qRT-PCR was performed using the SYBR green I PCR kit (Applied Biosystems, Foster City, CA, USA) in an ABI Prism 7900HT Sequence Detection System (Applied Biosystems). Each reaction contained $12.5 \mu \mathrm{l}$ SYBR green I PCR kit, $250 \mathrm{~nm}$ of reverse and forward primers and $5 \mu \mathrm{l}$ of cDNA. The total volume of the reaction was adjusted with water to $25 \mu \mathrm{l}$. PCR was performed in MicroAmp 96-well plates (Applied Biosystems) capped with MicroAmp optical adhesive seal. The reactions were incubated at $50^{\circ} \mathrm{C}$ for $2 \mathrm{~min}$, at $95^{\circ} \mathrm{C}$ for $10 \mathrm{~min}$, followed by 45 cycles at $95^{\circ} \mathrm{C}$ for $15 \mathrm{~s}$ and at $60^{\circ} \mathrm{C}$ for $1 \mathrm{~min}$. The PCR reactions were subjected to a heat dissociation protocol present in the ABI SDS 2.0 software (Applied Biosystems).

Cytokines were analyzed on Taqman Cytokine Gene Expression Plate I (Applied Biosystems) according to the manufacturer's instructions.

Serum cytokine measurements

The levels of TNF- $\alpha$, IFN- $\gamma$, IL- $1 \beta$ and IL-15 in serum were measured by the Pharmingen cytokine detection sets (BD Biosciences, San Jose, CA, USA) according to the manufacturer's instructions. The detection limits were for TNF- $\alpha 8 \mathrm{pg} / \mathrm{ml}, \mathrm{IFN}-\gamma 5 \mathrm{pg} / \mathrm{ml}, \mathrm{IL}-1 \beta 15 \mathrm{pg} / \mathrm{ml}$ and IL-15 $8 \mathrm{pg} / \mathrm{ml}$.

\section{Acknowledgements}

This study was supported by grants from the Swedish Medical Research Council, Samverkansnämnden, Västerbottens läns landsting, and the Medical Faculty, Umeå University, Umeå, Sweden, DARPA, Grant LN00A033 from Ministry of Education, Sport and Youth, Czech Republic.

\section{References}

1 Rubins KH, Hensley LE, Jahrling PB, Whitney AR, Geisbert TW, Huggins JW et al. The host response to smallpox: analysis of the gene expression program in peripheral blood cells in a 
nonhuman primate model. Proc Natl Acad Sci USA 2004; 101: 15190-15195.

2 Yu SL, Chen HW, Yang PC, Peck K, Tsai MH, Chen JJ et al. Differential gene expression in Gram-negative and Grampositive sepsis. Am J Respir Crit Care Med 2004; 169: 1135-1143.

3 Tärnvik A, Berglund L. Tularaemia. Eur Respir J 2003; 21: 361-373.

4 Dennis DT, Inglesby TV, Henderson DA, Bartlett JG, Ascher MS, Eitzen E et al. Tularemia as a biological weapon: medical and public health management. JAMA 2001; 285: 2763-2773.

5 Eliasson H, Sjöstedt A, Bäck E. Clinical use of a diagnostic PCR for Francisella tularensis in patients with suspected ulceroglandular tularaemia. Scand I Infect Dis 2005; 37: 833-837.

6 Syrjala H. Peripheral blood leukocyte counts, erythrocyte sedimentation rate and C-reactive protein in tularemia caused by the type B strain of Francisella tularensis. Infection 1986; 14: $51-54$.

7 Bolstad BM, Irizarry RA, Åstrand M, Speed TP. A comparison of normalization methods for high density oligonucleotide array data based on variance and bias. Bioinformatics 2003; 19: 185-193.

8 Lönnstedt IST. Replicated microarray data. Stat Sinica 2002; 12: $31-46$

9 Kovarova H, Halada P, Man P, Golovliov I, Krocova Z, Spacek J et al. Proteome study of Francisella tularensis live vaccine strain-containing phagosome in Bcg/Nramp1 congenic macrophages: resistant allele contributes to permissive environment and susceptibility to infection. Proteomics 2002; 2: 85-93.

10 Garin J, Diez R, Kieffer S, Dermine JF, Duclos S, Gagnon E et al. The phagosome proteome: insight into phagosome functions. I Cell Biol 2001; 152: 165-180.

11 Couillault C, Pujol N, Reboul J, Sabatier L, Guichou JF, Kohara $Y$ et al. TLR-independent control of innate immunity in Caenorhabditis elegans by the TIR domain adaptor protein TIR-1, an ortholog of human SARM. Nat Immunol 2004; 5: 488-494.

12 Song C, Jin B. TRAIL (CD253), a new member of the TNF superfamily. J Biol Regul Homeost Agents 2005; 19: 73-77.

13 Ledig S, Wagner S, Manns MP, Beil W, Athmann C. Role of the receptor-mediated apoptosis in Helicobacter pylori in gastric epithelial cells. Digestion 2004; 70: 178-186.

14 Ghayur T, Banerjee S, Hugunin M, Butler D, Herzog L, Carter A et al. Caspase-1 processes IFN-gamma-inducing factor and regulates LPS-induced IFN-gamma production. Nature 1997; 386: 619-623.

15 Hersh D, Monack DM, Smith MR, Ghori N, Falkow S, Zychlinsky A. The Salmonella invasin SipB induces macrophage apoptosis by binding to caspase-1. Proc Natl Acad Sci USA 1999; 96: 2396-2401.

16 Zhang L, Cui R, Cheng X, Du J. Antiapoptotic effect of serum and glucocorticoid-inducible protein kinase is mediated by novel mechanism ac. Cancer Res 2005; 65: 457-464.

17 Hitoshi Y, Lorens J, Kitada SI, Fisher J, LaBarge M, Ring HZ et al. Toso, a cell surface, specific regulator of Fas-induced apoptosis in T cells. Immunity 1998; 8: 461-471.

$18 \mathrm{Kim} \mathrm{CH}$. The greater chemotactic network for lymphocyte trafficking: chemokines and beyond. Curr Opin Hematol 2005; 12: 298-304.

19 Seitzer U, Kayser K, Hohn H, Entzian P, Wacker HH, Ploetz S et al. Reduced T-cell receptor CD3zeta-chain protein and sustained CD3epsilon expression at the site of mycobacterial infection. Immunology 2001; 104: 269-277.

20 Wisniewski HG, Vilcek J. Cytokine-induced gene expression at the crossroads of innate immunity, inflammation and fertility: TSG-6 and PTX3/TSG-14. Cytokine Growth Factor Rev 2004; 15: 129-146.

$21 \mathrm{Hu}$ SP, Harrison C, Xu K, Cornish CJ, Geczy CL. Induction of the chemotactic S100 protein, $\mathrm{CP}-10$, in monocyte/macrophages by lipopolysaccharide. Blood 1996; 87: 3919-3928.
22 Fitzgerald KA, Rowe DC, Golenbock DT. Endotoxin recognition and signal transduction by the TLR4/MD2-complex. Microbes Infect 2004; 6: 1361-1367.

23 Ritchie KJ, Hahn CS, Kim KI, Yan M, Rosario D, Li L et al. Role of ISG15 protease UBP43 (USP18) in innate immunity to viral infection. Nat Med 2004; 10: 1374-1378.

24 Werner ER, Werner-Felmayer G, Fuchs D, Hausen A, Reibnegger G, Yim JJ et al. Tetrahydrobiopterin biosynthetic activities in human macrophages, fibroblasts, THP-1, and T 24 cells. GTP-cyclohydrolase I is stimulated by interferongamma, and 6-pyruvoyl tetrahydropterin synthase and sepiapterin reductase are constitutively present. J Biol Chem 1990; 265: 3189-3192.

25 Gross SS, Levi R, Madera A, Park KH, Vane J, Hattori Y. Tetrahydrobiopterin synthesis is induced by LPS in vascular smooth muscle and is rate-limiting for nitric oxide production. Adv Exp Med Biol 1993; 338: 295-300.

26 Ihle JN. STATs: signal transducers and activators of transcription. Cell 1996; 84: 331-334.

27 Rouyez MC, Lestingi M, Charon M, Fichelson S, Buzyn A, Dusanter-Fourt I. IFN regulatory factor-2 cooperates with STAT1 to regulate transporter associated with antigen processing-1 promoter activity. J Immunol 2005; 174: 3948-3958.

28 Asmis R, Wang Y, Xu L, Kisgati M, Begley JG, Mieyal JJ. A novel thiol oxidation-based mechanism for adriamycin-induced cell injury in human macrophages. FASEB J 2005; 13: 1866-1868.

29 Lähteenmaki K, Edelman S, Korhonen TK. Bacterial metastasis: the host plasminogen system in bacterial invasion. Trends Microbiol 2005; 13: 79-85.

30 Nakagawa I, Nakata M, Kawabata S, Hamada S. Transcriptome analysis and gene expression profiles of early apoptosisrelated genes in Streptococcus pyogenes-infected epithelial cells. Cell Microbiol 2004; 6: 939-952.

31 Poquet Y, Kroca M, Halary F, Stenmark S, Peyrat MA, Bonneville $\mathrm{M}$ et al. Expansion of Vgamma9 Vdelta2 T cells is triggered by Francisella tularensis-derived phosphoantigens in tularemia but not after tularemia vaccination. Infect Immun 1998; 66: 2107-2114

32 Kroca M, Tärnvik A, Sjöstedt A. The proportion of circulating gammadelta $\mathrm{T}$ cells increases after the first week of onset of tularaemia and remains elevated for more than a year. Clin Exp Immunol 2000; 120: 280-284.

33 Zhang K, Zhao H. Assessing reliability of gene clusters from gene expression data. Funct Integr Genomics 2000; 1: 156-173.

34 Jenner RG, Young RA. Insights into host responses against pathogens from transcriptional profiling. Nat Rev Microbiol 2005; 3: 281-294.

35 Nagelkerke NJD. A note on a general definition of the coefficient of determination. Biometrika 1991; 78: 691-692.

36 Andersson H, Hartmanová B, Bäck E, Eliasson H, Landfors M, Rydén $\mathrm{P}$ et al. Transcriptional profiling of the peripheral blood response during tularemia. J Med Microbiol 2006; 55 263-271.

37 Conlan JW, Chen W, Shen H, Webb A, KuoLee R. Experimental tularemia in mice challenged by aerosol or intradermally with virulent strains of Francisella tularensis: bacteriologic and histopathologic studies. Microb Pathog 2003; 34: 239-248.

38 Tärnvik A, Sandström G, Löfgren S. Time of lymphocyte response after onset of tularemia and after tularemia vaccination. J Clin Microbiol 1979; 10: 854-860.

39 Sjöstedt A. Intracellular survival mechanisms of Francisella tularensis, a stealth pathogen. Microbes Infect 2005; 8: 561-567.

40 Lai XH, Sjöstedt A. Delineation of the molecular mechanisms of Francisella tularensis-induced apoptosis in murine macrophages. Infect Immun 2003; 71: 4642-4646.

41 Lai XH, Golovliov I, Sjöstedt A. Francisella tularensis induces cytopathogenicity and apoptosis in murine macrophages via a mechanism that requires intracellular bacterial multiplication. Infect Immun 2001; 69: 4691-4694. 
42 Telepnev M, Golovliov I, Grundström T, Tärnvik A, Sjöstedt A Francisella tularensis inhibits Toll-like receptor-mediated activation of intracellular signalling and secretion of TNFalpha and IL-1 from murine macrophages. Cell Microbiol 2003; 5: $41-51$.

43 Whitney AR, Diehn M, Popper SJ, Alizadeh AA, Boldrick JC, Relman DA et al. Individuality and variation in gene expression patterns in human blood. Proc Natl Acad Sci USA 2003; 100: 1896-1901.

44 Thach DC, Lin B, Walter E, Kruzelock R, Rowley RK, Tibbetts $\mathrm{C}$ et al. Assessment of two methods for handling blood in collection tubes with RNA stabilizing agent for surveillance of gene expression profiles with high density microarrays. J Immunol Methods 2003; 283: 269-279. 